\title{
Evaluation of related variables on endothelial progenitor cells in first transient ischemic attack
}

\author{
Rokhsareh Meamar ${ }^{1,2^{*}}$, Hamidreza Nikyar ${ }^{1}$, Ahmad Chitsaz ${ }^{3}$, Leila Dehghani ${ }^{4}$, Maryam Nasri $^{5}$ \\ 'Development Research Center, NajafAbad Branch, Islamic Azad University, Najafabad, Iran \\ ${ }^{2}$ Isfahan Clinical Toxicology Research Center, Isfahan University of Medical Sciences, Isfahan, Iran \\ ${ }^{3}$ Department of Neurology, Isfahan University of Medical Sciences, Isfahan, Iran \\ ${ }^{4}$ School of Advanced Technologies in Medicine, Shahid Beheshti University of Medical Sciences, Tehran, Iran \\ ${ }^{5}$ Grovemead Health Center, London, UK
}

*Corresponding Author: Rokhsareh Meamar, MD, Ph.D.; Development Research Center, NajafAbad Branch, Islamic Azad University, Najafabad, Iran and Isfahan clinical toxicology Research Center, Isfahan University of medical sciences, Isfahan, Iran. Email: meamar@pharm.mui.ac.ir

\begin{abstract}
Background and aims: In a transient ischemic attack (TIA), the activation of the endothelial progenitor cells (EPCs) has a prominent role in the formation of collateral vessels. The aim of this study was to evaluate the role of effective variables (e.g., hypertension, diabetes, hyperlipidemia, cardiovascular diseases, a family history of cardiovascular diseases, and statin therapy on the level of EPCs in TIA.

Methods: Thirty patients suffering from the first attack of TIA, without having a history of acute cerebral injury, surgery or trauma, and blood disorders were enrolled in this cross-sectional study. Then, flow cytometry was utilized to estimate the level of EPCs CD34 and CD309 and the results were evaluated based on the $t$ test or the Mann-Whitney test. Finally, the Pearson or Spearman correlation was used to establish the relationship between the variables. The level of $P<0.05$ was considered statistically significant in this study.

Results: The mean \pm SD number of CD309 in patients with hyperlipidemia was less than those with no hyperlipidemia $(3.35 \pm 2.77$ vs. $5.59 \pm 3.85, P=0.02)$ and diabetic patients had a significantly higher number of CD309 compared to non-diabetics $(6.14 \pm 4.89 \mathrm{vs}, 3.5 \pm$ $3.49, P=0.05)$. Conversely, the mean number of CD34 in groups with or without the studied variables was not statistically significant. The results further revealed that the average total of CD309 and CD34 was significantly lower in patients with hyperlipidemia as compared to those with no sign of hyperlipidemia $(9.44 \pm 3.05$ vs. $6.67 \pm 4.6, P=0.02)$. Using logistic regression, the intended variables demonstrated no significant effects on endothelial cells, and the relationship between age and the number of progenitor cells was not significant.

Conclusion: In our study, only hyperlipidemia acted as a factor influencing the numbers of EPCs. Therefore, more studies with larger sample sizes are required to discover the role of these variables on progenitor cells in TIA.

Keywords: Transient ischemic attack; Endothelial progenitor cell; Risk factors
\end{abstract}

Received: 29 October 2017, Accepted: 17 June 2018, ePublished: 30 August 2019

\section{Introduction}

Endothelial injury and atherosclerosis heavily contribute to cerebrovascular and cardiovascular diseases, (1) and ischemia promotes the migration of endothelial progenitor cells (EPCs) to initiate generating new vessels after myocardial infarction (2).

The important role of these cells is established in the vessel repair and generation in the brain after an injury caused by the ischemia (2). These cells originate from the bone marrow and each one of them expresses a marker that differentiates it from the other. For example, EPCs CD34 are the progenitor cells and the endothelial marker CD309 or VEGFR-2 stimulates the growth of the endothelial cells in the vessels (3). The role of EPCs in the repair of vessels after brain ischemia has been confirmed in previous research and these cells are recognized as a factor that promotes neovascularization and may stimulate the activity of the metalloproteinase matrix (4). Clearly, the decreased size of the infarct site after acute ischemia is associated with an increase in the number of circulating EPCs (5).

Transient ischemic attack (TIA) is regarded as a focal ischemic attack that, as opposed to the cerebrovascular accident (CVA), lasts less than an hour and demonstrates no necrosis of the tissues. In addition, TIA is a predicting factor for future CVAs, because it can be followed by a CVA (6) and its risk factors include age, gender, family history, smoking, hypertension (HTN), and diabetes (7). Some factors have a protective effect such as statin usage, which reduces atherosclerosis and, therefore, decreases the risk of TIA (8). The injured endothelium causes microand macrovascular diseases in diabetic patients and is considered as a factor for cardiac events in this population (9). Considering the above-mentioned explanations, the

(C) 2019 The Author(s); Published by Shahrekord University of Medical Sciences. This is an open-access article distributed under the terms of the Creative Commons Attribution License (http://creativecommons.org/licenses/by/4.0), which permits unrestricted use, distribution, and reproduction in any medium, provided the original work is properly cited. 
current study attempted to investigate the effect of different variables (e.g., hypertension, diabetes, hyperlipidemia, cardiovascular diseases, a family history of cardiovascular diseases, and statin therapy) on the number of EPCs in TIA.

\section{Materials and Methods}

The materials and sources of the purchase are listed below.

- Antibodies and Calibur flow cytometer (Becton Dickinson, San Jose, CA, USA);

- $\quad$ Lysis solution (Dako, Glostrup, Denmark);

- $\quad$ Fetal calf serum (HyClone, Logan, UT, USA);

- Cell Quest Software and stain buffer (BD Biosciences, San Diego, CA, USA);

- $\quad$ Fc-blocking agent (Miltenyi Biotech);

- CD34-FITC and CD309-PE (Miltenyi Biotech).

A number of 30 participants suffering from their first TIA were included in the present cross-sectional study, who were selected based on TOAST (Trial of ORG 10172 in Acute Stroke Treatment) criteria (10) and admitted to the emergency department of Al-Zahra hospital between December 2012 and October 2013. The exclusion criteria included being under 18 or above 80 years of age, having a history of intracranial hemorrhage, surgery, or trauma during the last three months, as well as having liver dysfunction, hematological abnormalities, renal failure, malignancy, acute or chronic inflammatory conditions, atrial fibrillation, and also being among the angiotensinconverting medication users.

The obtained history included variables related to diabetes (fasting blood sugar above $126 \mathrm{mg} / \mathrm{dL}$ ), hypertension (BP $>/ 140 / 90$ ), hyperlipidemia (total cholesterol $>/ 240 \mathrm{mg} / \mathrm{dL}$ or TG $>/ 200$ ), and smoking (a minimum of five cigarettes a day for one year), along with a family history of CHD including angina and myocardial infarction and sudden cardiac death in the immediate family.

Blood sampling and the assessment of the number of circulating endothelial progenitor cells by flowcytometry Blood samples were obtained 24 hours after TIA and processed two hours afterward. In addition, the EPCs in unselected peripheral cells and their level were measured by phenotypic analysis and flow cytometry, respectively. Fifty microliters of ethylenediamine tetraacetic acid-anticoagulated blood was incubated for 30 minutes at $4{ }^{\circ} \mathrm{C}$, along with $20 \mu \mathrm{L}$ of fluorescein isothiocyanate (FITC)-conjugated anti-CD34 and 10 $\mu \mathrm{L}$ of phycoerythrin (PE)-conjugated anti-CD309. Appropriate isotype controls were used for each staining process. One milliliter of lysis solution was added for 5 minutes at room temperature, and then the samples were centrifuged and pellets were re-suspended in $300 \mu \mathrm{L}$ of phosphate buffered saline. Two sets of 105 cells were obtained by a FACS (fluorescence-activated cell sorting) Caliber flow cytometer and analyzed as well. The number of dead/apoptotic cells was negligible, therefore, the cellular debris in a side-scatter/forward scatter dot plot was excluded from the analysis. CD34+ cells were electronically gated, followed by evaluating the percentage of the cells co-expressing CD309. A minimum of 20000 events was obtained for each sample. Further, the presence of at least $0.03 \%$ nucleated cells co-expressing three antigens over the background fluorescence was defined by flow cytometry as EPC detection, and the results were expressed as a percentage of CD34+ cell that co-expressed CD309. The absolute number of CD34+ CD309+ cells was also calculated based on the peripheral nucleated blood count, and the expression of cell surface antigens was determined by two-color immunofluorescence staining. Furthermore, two sets of 106 cells were incubated in a buffer containing $2 \%$ bovine serum albumin with $20 \mu$ Fc-blocking agent for 10 minutes at $25^{\circ} \mathrm{C}$. The cells were then incubated for 30 minutes at $4{ }^{\circ} \mathrm{C}$ with $20 \mu \mathrm{L}$ of CD34-FITC and CD309-PE (a total volume of $200 \mu \mathrm{L}$ ) and washed twice before re-suspension in $400 \mu \mathrm{L}$ stain buffer. Eventually, FACS analysis was performed in triplicate for each sample.

\section{Data Analysis}

The mean, standard deviation, median, and range were defined based on the study purposes and Shapiro-Wilk test was used to determine the normal distribution of data. Then, the statistical relation between CD34 and CD309 separately and their total relationship with the intended variables were estimated by the independent $t$ test and Mann-Whitney test and the final analysis was performed by the logistic regression model. Similarly, Pearson and Spearman tests were utilized to investigate the relationship between EPC and age. Eventually, SPSS 21 (IBM USA) was used and $P<0.05$ was considered statistically significant.

Results

The mean \pm standard deviation (SD) of the age of the patients with the first TIA was $63 \pm 10.5$. In general, 8 $(26 \%)$ and $22(74 \%)$ of the patients were females and males, respectively. Based on the data in Table 1,12 (44\%) patients had coronary heart disease (CHD) and 22 (74\%) of them had HTN, and the mean of CD309 in the CHD patients $(P=0.89)$ and HTN $(P=0.22)$ showed no statistically significant difference with healthy participants.

Moreover, the mean number of CD309 in individuals with a family history of CHD ( $n=16,53 \%)$ was not statistically significant as compared to those with no history of CHD $(P=0.93)$. Likewise, no statistical difference was observed in the CD309 of patients who used statins $(\mathrm{n}=22,73 \%)$ compared to those who consumed no statins $(P=0.32)$. Additionally, CD309 was lower in those who had a high serum lipid $(n=9,30 \%)$ compared to those with normal lipids, indicating a meaningful difference $(P=0.02)$. The CD309 count in those who had diabetes mellitus (DM) was higher compared to those with no DM 
Table 1. CD309 numbers based on the related variables

\begin{tabular}{|c|c|c|c|c|}
\hline & & \multicolumn{2}{|r|}{ CD309 } & \multirow{2}{*}{$P$-value } \\
\hline & & Mean \pm SD & Median (Interquartile Range) & \\
\hline \multirow{2}{*}{ Blood pressure } & Yes $(n=22)$ & $4.04 \pm 3.23$ & $3.11(2.02,4.70)$ & \multirow{2}{*}{0.22} \\
\hline & No $(n=8)$ & $3.99 \pm 3.54$ & $1.84(0.47,5.59)$ & \\
\hline \multirow{2}{*}{ Smoking } & Yes $(n=10)$ & $2.79 \pm 2.76$ & $2.55(0.82,2.95)$ & \multirow{2}{*}{0.14} \\
\hline & No $(n=20)$ & $4.64 \pm 4.24$ & $3.13(1.96,5.71)$ & \\
\hline \multirow{2}{*}{ Diabetes } & Yes $(n=6)$ & $6.14 \pm 4.89$ & $5.52(3.12,6.78)$ & \multirow{2}{*}{0.05} \\
\hline & No $(n=24)$ & $3.5 \pm 3.49$ & $2.55(1.83,4.3)$ & \\
\hline \multirow{2}{*}{ Cardiovascular disease } & Yes $(n=12)$ & $4.82 \pm 4.32$ & $2.61(1.4,5.74)$ & \multirow{2}{*}{0.89} \\
\hline & No $(n=18)$ & $3.5 \pm 2.54$ & $2.97(1.90,4.30)$ & \\
\hline \multirow{2}{*}{ Family history of cardiovascular disease } & Yes $(n=16)$ & $4.31 \pm 3.53$ & $2.89(1.9,4.31)$ & \multirow{2}{*}{0.93} \\
\hline & No $(n=14)$ & $3.69 \pm 3.08$ & $2.97(0.85,6.77)$ & \\
\hline \multirow{2}{*}{ Hyperlipidemia } & Yes $(n=9)$ & $3.35 \pm 2.77$ & $2.12(3.12,6.77)$ & \multirow{2}{*}{0.02} \\
\hline & No $(n=21)$ & $5.59 \pm 3.85$ & $4.32(0.88,3.14)$ & \\
\hline \multirow{2}{*}{ Statin usage } & Yes $(n=22)$ & $4.39 \pm 4.12$ & $3.03(2.02,4.70)$ & \multirow{2}{*}{0.32} \\
\hline & No $(n=8)$ & $3.01 \pm 2.05$ & $2.37(0.50,4.96)$ & \\
\hline
\end{tabular}

a Mann-Whitney $U$ test.

(n=6, 20\% vs. $\mathrm{n}=22,80 \%, P=0.05)$. This was clinically significant as well.

However, the mean count of CD34 was not statistically different in any of the groups containing the study variables (Table 2). Table 3 shows that the total of CD309 and CD34 in individuals with high serum lipids was lower as compared to those who had normal lipids $(P=0.02)$. In addition, the other variables such as $\operatorname{DM}(P=0.11)$ and HTN $(P=0.89)$ revealed no meaningful effect on the total of CD309 and CD34. Based on the advanced analysis of the logistic regression, the variables showed an effect on the level of the endothelial cells as well (Table 4).

The relationship between age and the number of endothelial factors demonstrated a weak and inverse effect $(P=0.46, r=-0.138)$. Similarly, a positive weak effect was observed between CD309 $(P=0.16, \mathrm{r}=0.26)$ and age $\mathrm{CD} 34+\mathrm{Cd} 309(P=0.23, \mathrm{r}=0.22)$ that was not meaningful.

\section{Discussion}

The current study examined the effect of the studied variables (i.e., protective and risk factors) on the number of EPCs in TIA, and it was found that CD309 count and the total of CD309 and CD34 count were statistically lower in individuals with high lipids but CD309 was higher in people with DM. In a previous study, increased circulatory EPC levels were detected in the first TIA attack as compared to the control. However, this failed to increase the incidence of cerebrovascular disease and the level of EPC was not considered as a predictive factor in the study population (11). This study aimed to find the role of TIA

Table 2. CD34 numbers based on the Intended variables

\begin{tabular}{|c|c|c|c|c|}
\hline & & \multicolumn{2}{|r|}{ CD34 } & \multirow{2}{*}{$\boldsymbol{P}$-value } \\
\hline & & Mean \pm SD & Median (Interquartile Range) & \\
\hline \multirow{2}{*}{ Blood pressure } & Yes $(n=22)$ & $3.57 \pm 2.0$ & $3.2(2.06,5.35)$ & \multirow{2}{*}{0.57} \\
\hline & No $(n=8)$ & $3.27 \pm 2.13$ & $2.68(1.47,5.47)$ & \\
\hline \multirow{2}{*}{ Smoking } & Yes $(n=10)$ & $3.59 \pm 2.17$ & $3.68(2.01,5.65)$ & \multirow{2}{*}{0.94} \\
\hline & No $(n=20)$ & $3.43 \pm 1.97$ & $3.2(2.06,5.31)$ & \\
\hline \multirow{2}{*}{ Diabetes } & Yes $(n=6)$ & $3.86 \pm 2.12$ & $3.31(3.13,5.68)$ & \multirow{2}{*}{0.53} \\
\hline & No $(n=24)$ & $3.39 \pm 2.01$ & $3.15(2.03,5.34)$ & \\
\hline \multirow{2}{*}{ Cardiovascular disease } & Yes $(n=12)$ & $3.36 \pm 1.85$ & $3.22(2.04,5.32)$ & \multirow{2}{*}{0.88} \\
\hline & No $(n=18)$ & $3.57 \pm 2.15$ & $3.19(2.04,5.60)$ & \\
\hline \multirow{2}{*}{ Family history of cardiovascular disease } & Yes $(n=16)$ & $3.67 \pm 1.88$ & $3.46(2.05,5.34)$ & \multirow{2}{*}{0.57} \\
\hline & No $(n=14)$ & $3.27 \pm 2.19$ & $2.57(2.01,5.68)$ & \\
\hline \multirow{2}{*}{ Hyperlipidemia } & Yes $(n=9)$ & $3.85 \pm 2.23$ & $3.25(2.08,6.08)$ & \multirow{2}{*}{0.42} \\
\hline & No $(n=21)$ & $3.33 \pm 1.94$ & $3.15(2.01,5.32)$ & \\
\hline \multirow{2}{*}{ Statin usage } & Yes $(n=22)$ & $3.76 \pm 1.94$ & $3.27(2.08,5.35)$ & \multirow{2}{*}{0.21} \\
\hline & No $(n=8)$ & $2.74 \pm 2.11$ & $2.04(0.92,4.56)$ & \\
\hline
\end{tabular}

a Mann-Whitney U test. 


\begin{tabular}{|c|c|c|c|c|}
\hline & & \multicolumn{2}{|r|}{ CD34+CD309 } & \multirow{2}{*}{$P$-value ${ }^{a}$} \\
\hline & & Mean \pm SD & Median (Interquartile Range) & \\
\hline \multirow{2}{*}{ Blood pressure } & Yes $(n=22)$ & $7.6 \pm 3.45$ & $7.48(5.15,9.97)$ & \multirow{2}{*}{0.89} \\
\hline & No $(n=8)$ & $7.25 \pm 6.48$ & 4.88 (2.53 to 11.43$)$ & \\
\hline \multirow{2}{*}{ Smoking } & Yes $(n=10)$ & $6.38 \pm 4.4$ & $5.54(2.91,8.47)$ & \multirow{2}{*}{0.32} \\
\hline & No $(n=20)$ & $8.06 \pm 4.3$ & $7.57(5.16,10.0)$ & \\
\hline \multirow{2}{*}{ Diabetes } & Yes $(n=6)$ & $9.99 \pm 4.02$ & $9.91(7.79,12.46)$ & \multirow{2}{*}{0.11} \\
\hline & No $(n=24)$ & $6.88 \pm 4.26$ & $6.2(3.87,9.41)$ & \\
\hline \multirow{2}{*}{ Cardiovascular disease } & Yes $(n=12)$ & $8.17 \pm 5.46$ & $7.47(3.47,11.62)$ & \multirow{2}{*}{0.50} \\
\hline & No $(n=18)$ & $7.06 \pm 3.5$ & $6.84(5.15,9.85)$ & \\
\hline \multirow{2}{*}{ Family history of cardiovascular disease } & Yes $(n=16)$ & $7.97 \pm 4.33$ & $7.33(5.08,9.05)$ & \multirow{2}{*}{0.53} \\
\hline & No $(n=14)$ & $6.97 \pm 4.44$ & $7.19(2.91,10.02)$ & \\
\hline \multirow{2}{*}{ Hyperlipidemia } & Yes $(n=9)$ & $6.67 \pm 4.6$ & $5.17(7.79,10.02)$ & \multirow{2}{*}{0.02} \\
\hline & No $(n=21)$ & $9.44 \pm 3.05$ & $9.85(3.68,8.47)$ & \\
\hline \multirow{2}{*}{ Statin usage } & Yes $(n=22)$ & $8.15 \pm 4.03$ & $7.71(5.18,9.97)$ & \multirow{2}{*}{0.18} \\
\hline & No $(n=8)$ & $5.73 \pm 4.93$ & $3.87(2.53,8.81)$ & \\
\hline
\end{tabular}

a Independent sample $t$ test.

Table 4. Evaluation of the effect of the related variables on the number of EPCs using logistic regression model

\begin{tabular}{|c|c|c|c|c|c|c|}
\hline & \multicolumn{2}{|c|}{ CD34+CD309 } & \multicolumn{2}{|c|}{ CD34 } & \multicolumn{2}{|c|}{ CD309 } \\
\hline & $\beta(\mathrm{SD})$ & $P$-value & $\beta(\mathrm{SD})$ & $P$-value & $\beta(\mathrm{SD})$ & $P$-value \\
\hline Blood pressure & $-0.02(0.41)$ & 0.91 & $0.15(0.44)$ & 0.61 & $0.05(0.7)$ & 0.82 \\
\hline Smoking & $0.08(0.39)$ & 0.84 & $0.17(0.42)$ & 0.54 & $-0.13(0.67)$ & 0.61 \\
\hline Diabetes & $0.17(0.35)$ & 0.39 & $0.03(0.38)$ & 0.89 & $0.12(0.60)$ & 0.55 \\
\hline Hyperlipidemia & $-0.25(0.33)$ & 0.25 & $0.13(0.36)$ & 0.58 & $-1.3(0.86)$ & 0.13 \\
\hline
\end{tabular}

Abbreviations: EPCs, Endothelial progenitor cells; SD, Standard deviation.

risk factors in the level of these cells.

Endothelial cell injury is viewed as one of the most important factors causing atherosclerosis (1), and the dysfunction of endothelial cells is a predictive factor for cardiovascular (12) and cerebrovascular diseases (13). Similarly, atherosclerosis and age are the most significant risk factors which reduce the number of circulating EPCs (14). Hypercholesterolemia (15), smoking, and diabetes are other risk factors, while statin therapy (16) and physical exercise (17) have a positive effect on the primary and secondary prevention of atherosclerosis.

The process of atherosclerosis could partially be attributed to the accumulation of lipids in the vascular walls. Epidemiology and observational studies reported a causal relationship between serum lipids and high cholesterol (LDL-C) with atherosclerosis (18). Hill et al showed that the EPCs decreased in healthy subjects with cardiovascular risk factors, while only cholesterol remained a significant factor when adjusted for age and individual risk factors such as cholesterol, HTN, and MD (19). Similarly, Chen et al concluded that the number of EPCs in people with high cholesterol was lower than that in the control group and this parameter had a negative relationship with total cholesterol and LDL-C (20). Although a reduction was observed in the number of endothelial precursor cells in people with high cholesterol, this observation was less significant in the subgroup with HDL-C (21), which was confirmed by the findings of our study. In TIA, sufferers who have higher serum lipids have a reduced number of EPCs as compared to people with normal serum lipids.

Werner et al discovered that a decline in endothelial precursor cells was related to increased LDL-C, and statin therapy was related to an increase in these cells (22). It appears that the effect of statin therapy on the cells is independent of their effect on reducing LDL-C and the preventative effect of this medication is probably through stimulating the EPCs. The results of our study showed the increased number of the cells, while the increase was negligible, which may be attributed to the small sample size.

The mechanism of endothelial injury by high blood sugar is well-established, and it is known that endothelial injury results in micro- and macrovascular complications over time. Some researchers confirmed the relationship between EPCs and endothelial injury in diabetic patients. For example, Loomans et al demonstrated that the number of EPCs decreased in diabetes and had an inverse effect on glycated hemoglobin (HbA1C) (23). In another study, the number of EPCs represented a reduction in people suffering from diabetes type 2 and was related to their 


\section{HbA1C (24)}

A decrease in the number of CD34 progenitor cells was observed in diabetic patients and those with glucose intolerance as well. The The level of progenitor cells continue continues to decrease as progression is detected in the disease. The increase in the levels of EPC leads to the angiogenesis of the retina and augmentation, and thus the generation of EPCs is observed type 1 (25) and type 2 (26) in diabetic patients with proliferative retinopathy. Interestingly, in previous research, dysregulation in migration and transport was reported with an increase in the number of progenitor endothelial cells, and this pattern of increase in dysfunctional cells was observed in type 1 and 2 diabetic patients (27). In our study, the number of EPCs, especially CD309, was higher in those with DM compared to those with no symptom of diabetes. One explanation would be that our patients were in the advanced stages of the disease, which may explain the angiogenesis and dysfunctional state of the cells.

Diabetes is suggested as a facilitator for endothelial injury, and therapy with such medications as metformin or glitazone can augment EPCs independent of blood sugar control $(28,29)$. All our patients were using metformin or pioglitazone. This may account for the increasing level of these cells in these patients and the increased reserve in diabetic patients. However, more studies are required to focus on diabetic patients with TIA to establish the role and level of these cells.

Although other variables that could potentially affect EPCs were studied, there was no significant difference in our study in this regard. Mobarrez et al explained that cigarettes are an influential tractor on the number of EPCs (30). Similarly, Yeoh et al indicated that the level of these cells in smokers who suffered from cardiovascular diseases was extremely lower compared to non-smokers in the control group suffering from cardiovascular diseases (31). The number of these cells had also a positive relationship with the number of cigarettes. However, the level of the cells was lower in smokers of the current study compared to non-smokers while it was not significant, which could be attributed to the small sample of people with TIA.

Despite the epidemiology and physiological importance of HTN, previous studies obtained controversial results as regards to the relationship between HTN and EPCs. For instance, Hill et al found a strong relationship between the level of these cells and the Framingham calculated risk, while discovering an inverse relationship between these cells and HTN. However, this negative relationship disappeared when adjusted for age (32). In another study, the number of CD34 was higher in people with HTN and cardiovascular diseases compared to those with normal blood pressure (33). On the other hand, Delva et al studied the function and level of EPCs in individuals with HTN and found no significant statistical difference between the number of EPCs in these subjects and the control group
(34). In addition, there was no difference in TIA patients in HTN compared to the control group in our study.

Various events happen in the organs with age; therefore, advanced age is a critical risk factor for vascular diseases and researchers found an inverse relationship between age and the number of progenitor cells (35). Thus, it is concluded that those who have a higher number of these cells (CD34) during their younger life have a higher probability to live longer. Dimmeler and Vasa-Nicotera concluded that sensitivity to apoptosis increases with age, and thus it affects the survival of EPCs (36). In ancestor cells, many factors for example reduction in antioxidant defence or shortened telomere stage are responsible. EPCs are dysfunctional in regeneration, survival, and migration in older adults. In our study, an inverse relationship was found between CD34 and age, but it was not statistically significant.

Based on the results of our study, serum lipids were considered as an effective factor in the reduction of EPCs. Further, the mean number of CD309 in people with higher serum lipids was lower compared to people with normal serum lipids, and CD309 demonstrated a statistically significant increase in diabetic patients. However, CD34 level showed no meaningful relationship with any of the variables. In contrast, the mean total of CD309 and CD34 was significantly lower in people with increased serum lipids when compared to those with no high serum lipids. Accordingly, further studies with larger sample size are required for evaluating the factors that could affect endothelial cells in a TIA event.

Conflict of interests

None.

Ethical considerations

The study was ethically approved (under the ethical code of 1509507130038) and a consent form was obtained from all participants.

\section{Acknowledgments}

This study was extracted from the research project number 1509507130038 that was registered in the Islamic Azad University of Najaf Abad. We are grateful to the colleagues at the Neurosciences Research Department and the staff of the genetics laboratory in Alzahra hospital for contributing to this study.

\section{References}

1. Wang X, Chappell FM, Valdes Hernandez M, Lowe G, Rumley A, Shuler K, et al. Endothelial Function, Inflammation, Thrombosis, and Basal Ganglia Perivascular Spaces in Patients with Stroke. J Stroke Cerebrovasc Dis. 2016;25(12):2925-31. doi: 10.1016/j.jstrokecerebrovasdis.2016.08.007.

2. Nour M, Scalzo F, Liebeskind DS. Ischemia-reperfusion injury in stroke. Interv Neurol. 2013;1(3-4):185-99. doi: 10.1159/000353125.

3. Farhan MA, Azad AK, Touret N, Murray AG. FGD5 regulates VEGF receptor-2 coupling to PI3 kinase and receptor recycling. Arterioscler Thromb Vasc Biol. 2017;37(12):230110. doi: 10.1161/atvbaha.117.309978.

4. Werner $N$, Junk S, Laufs $U$, Link A, Walenta K, Bohm M, 
et al. Intravenous transfusion of endothelial progenitor cells reduces neointima formation after vascular injury. Circ Res. 2003;93(2):e17-24. doi: 10.1161/01. res.0000083812.30141.74.

5. Pías-Peleteiro J, Pérez-Mato M, López-Arias E, RodríguezYáñez M, Blanco M, Campos F, et al. Increased endothelial progenitor cell levels are associated with good outcome in intracerebral hemorrhage. Sci Rep. 2016;6:28724. doi: 10.1038/srep28724.

6. van Rooy MJ, Pretorius E. Metabolic syndrome, platelet activation and the development of transient ischemic attack or thromboembolic stroke. Thromb Res. 2015;135(3):434-42. doi: 10.1016/j.thromres.2014.12.030.

7. Giles MF, Rothwell PM. Risk prediction after TIA: the ABCD system and other methods. Geriatrics. 2008;63(10):10-3, 6.

8. Khare S. Risk factors of transient ischemic attack: An overview. J Midlife Health. 2016;7(1):2-7. doi: 10.4103/09767800.179166.

9. Wils J, Favre J, Bellien J. Modulating putative endothelial progenitor cells for the treatment of endothelial dysfunction and cardiovascular complications in diabetes. Pharmacol Ther. 2017;170:98-115. doi: 10.1016/j.pharmthera.2016.10.014.

10. Amort M, Fluri F, Weisskopf F, Gensicke H, Bonati LH, Lyrer $\mathrm{PA}$, et al. Etiological classifications of transient ischemic attacks: subtype classification by TOAST, CCS and ASCO-a pilot study. Cerebrovasc Dis. 2012;33(6):508-16. doi: 10.1159/000337236.

11. Meamar R, Nikyar H, Dehghani L, Talebi M, Dehghani M, Ghasemi $M$, et al. The role of endothelial progenitor cells in transient ischemic attack patients for future cerebrovascular events. Journal of Research in Medical Sciences. 2016;21(1):47. doi: 10.4103/1735-1995.183995.

12. Widmer RJ, Lerman A. Endothelial dysfunction and cardiovascular disease. Glob Cardiol Sci Pract. 2014;2014(3):291-308. doi: 10.5339/gcsp.2014.43.

13. Urso C, Caimi G. [Oxidative stress and endothelial dysfunction]. Minerva Med. 2011;102(1):59-77.

14. Schmidt-Lucke C, Rössig L, Fichtlscherer S, Vasa M, Britten M, Kämper $U$, et al. Reduced number of circulating endothelial progenitor cells predicts future cardiovascular events: proof of concept for the clinical importance of endogenous vascular repair. Circulation. 2005;111(22):2981-7. doi: 10.1161/ circulationaha.104.504340.

15. Aragona CO, Imbalzano E, Mamone F, Cairo V, Lo Gullo A, D'Ascola A, et al. Endothelial progenitor cells for diagnosis and prognosis in cardiovascular disease. Stem Cells Int. 2016;2016:8043792. doi: 10.1155/2016/8043792.

16. Sobrino T, Blanco $M$, Pérez-Mato $M$, Rodríguez-Yáñez $M$, Castillo J. Increased levels of circulating endothelial progenitor cells in patients with ischaemic stroke treated with statins during acute phase. Eur J Neurol. 2012;19(12):1539-46. doi: 10.1111/j.1468-1331.2012.03770.x.

17. Ribeiro F, Ribeiro IP, Goncalves AC, Alves AJ, Melo E, Fernandes $R$, et al. Effects of resistance exercise on endothelial progenitor cell mobilization in women. Sci Rep. 2017;7(1):17880. doi: 10.1038/s41598-017-18156-6.

18. Gao S, Liu J. Association between circulating oxidized lowdensity lipoprotein and atherosclerotic cardiovascular disease. Chronic Dis Transl Med. 2017;3(2):89-94. doi: 10.1016/j. cdtm.2017.02.008.

19. Hill JM, Zalos G, Halcox JP, Schenke WH, Waclawiw MA, Quyyumi AA, et al. Circulating endothelial progenitor cells, vascular function, and cardiovascular risk. N Engl J Med. 2003;348(7):593-600. doi: 10.1056/NEJMoa022287.

20. Chen JZ, Zhang FR, Tao QM, Wang XX, Zhu JH, Zhu JH. Number and activity of endothelial progenitor cells from peripheral blood in patients with hypercholesterolaemia. Clin Sci (Lond). 2004;107(3):273-80. doi: 10.1042/CS20030389.

21. Rossi F, Bertone C, Montanile F, Miglietta F, Lubrano C, Gandini
L, et al. HDL cholesterol is a strong determinant of endothelial progenitor cells in hypercholesterolemic subjects. Microvasc Res. 2010;80(2):274-9. doi: 10.1016/j.mvr.2010.05.003.

22. Werner N, Kosiol S, Schiegl T, Ahlers P, Walenta K, Link A, et al. Circulating endothelial progenitor cells and cardiovascular outcomes. N Engl J Med. 2005;353(10):999-1007. doi: 10.1056/NEJMoa043814.

23. Loomans CJ, de Koning EJ, Staal FJ, Rookmaaker MB, Verseyden $\mathrm{C}$, de Boer $\mathrm{HC}$, et al. Endothelial progenitor cell dysfunction: a novel concept in the pathogenesis of vascular complications of type 1 diabetes. Diabetes. 2004;53(1):195-9. doi: 10.2337/diabetes.53.1.195.

24. Georgescu A. Vascular dysfunction in diabetes: The endothelial progenitor cells as new therapeutic strategy. World J Diabetes. 2011;2(6):92-7. doi: 10.4239/wjd.v2.i6.92.

25. Asnaghi V, Lattanzio R, Mazzolari G, Pastore MR, Ramoni A, Maestroni A, et al. Increased clonogenic potential of circulating endothelial progenitor cells in patients with type 1 diabetes and proliferative retinopathy. Diabetologia. 2006;49(5):1109-11. doi: 10.1007/s00125-006-0180-0.

26. Brunner S, Hoellerl F, Schmid-Kubista KE, Zeiler F, Schernthaner G, Binder S, et al. Circulating angiopoietic cells and diabetic retinopathy in type 2 diabetes mellitus, with or without macrovascular disease. Invest Ophthalmol Vis Sci. 2011;52(7):4655-62. doi: 10.1167/iovs.10-6520.

27. Yu CG, Zhang N, Yuan SS, Ma Y, Yang LY, Feng YM, et al. Endothelial progenitor cells in diabetic microvascular complications: friends or foes? Stem Cells Int. 2016;2016:1803989. doi: 10.1155/2016/1803989.

28. Yu JW, Deng YP, Han X, Ren GF, Cai J, Jiang GJ. Metformin improves the angiogenic functions of endothelial progenitor cells via activating AMPK/eNOS pathway in diabetic mice. Cardiovasc Diabetol. 2016;15:88. doi: 10.1186/s12933-0160408-3.

29. Ku YH, Cho BJ, Kim MJ, Lim S, Park YJ, Jang HC, et al. Rosiglitazone increases endothelial cell migration and vascular permeability through Akt phosphorylation. BMC Pharmacol Toxicol. 2017;18(1):62. doi: 10.1186/s40360-0170169-y.

30. Mobarrez F, Antoniewicz L, Bosson JA, Kuhl J, Pisetsky DS, Lundback $M$. The effects of smoking on levels of endothelial progenitor cells and microparticles in the blood of healthy volunteers. PLoS One. 2014;9(2):e90314. doi: 10.1371/ journal.pone.0090314.

31. Yue WS, Wang $M$, Yan $G H$, Yiu $K H$, Yin $L$, Lee $S W$, et al. Smoking is associated with depletion of circulating endothelial progenitor cells and elevated pulmonary artery systolic pressure in patients with coronary artery disease. Am J Cardiol. 2010;106(9):1248-54. doi: 10.1016/j.amjcard.2010.06.045.

32. Hill JM, Zalos G, Halcox JP, Schenke WH, Waclawiw MA, Quyyumi AA, et al. Circulating endothelial progenitor cells, vascular function, and cardiovascular risk. N Engl J Med. 2003;348(7):593-600. doi: 10.1056/NEJMoa022287.

33. Mandraffino G, Imbalzano E, Sardo MA, D'Ascola A, Mamone F, Lo Gullo A, et al. Circulating progenitor cells in hypertensive patients with different degrees of cardiovascular involvement. J Hum Hypertens. 2014;28(9):543-50. doi: 10.1038/jhh.2014.7

34. Delva P, Degan M, Vallerio P, Arosio E, Minuz P, Amen G, et al. Endothelial progenitor cells in patients with essential hypertension. J Hypertens. 2007;25(1):127-32. doi: 10.1097/ HJH.0b013e3280109271.

35. Jie KE, Goossens MH, van Oostrom O, Lilien MR, Verhaar MC. Circulating endothelial progenitor cell levels are higher during childhood than in adult life. Atherosclerosis. 2009;202(2):3457. doi: 10.1016/j.atherosclerosis.2008.05.012.

36. Dimmeler S, Vasa-Nicotera M. Aging of progenitor cells: limitation for regenerative capacity? J Am Coll Cardiol. 2003;42(12):2081-2. doi: 10.1016/j.jacc.2003.09.016. 